Medicina Experimentalis 1962;7(suppl 1):195-197

\title{
Noms et adresses des participants français
}

Priv.-Doz. Dr. F.-J. M. Winzenried

Oberarzt der Psychiatrischen und Nervenklinik der Universität Hamburg

Prof. Dr. Dr. W. Wirth Pharmakol. Laboratorium der Farbenfabriken Bayer, Elberfeld

Dr. G. Zawuski Nervenklinik der Universität Köln, Köln-Lindenthal

Dr. H. U. Ziolko Psychiatrische und Neurologische Klinik der Freien Universität

Berlin, Berlin-Charlottenburg

Liste des participants français

Dr P. Balvet Médecin chef, Hôpital psychiatrique du Vinatier, Bron (Rhone)

Dr M. Baudry Médecin chef, Hôpital psychiatrique de la Timone, Boulevard

Bailie, Marseille (Bouches-du-Rhône)

Dr Ch. Berthier Médecin chef, Hôpital psychiatrique du Vinatier, Bron (Rhone)

Dr H. Bonnet Médecin chef, Hôpital psychiatrique de Sainte-Marie, Montredon,

Le Puy (Haute-Loire)

Dr L. Bourrat ler Assistant du Service de clinique neuro-psychiatrique et

d'Hygiène mentale, Hôpital Edouard Herriot, Lyon (Rhone)

Dr P. Broussolle Médecin chef, Hôpital psychiatrique du Vinatier, Bron (Rhone)

Dr P. Carrier Ex-chef de Clinique neuro-psychiatrique, 244, Route de Vienne,

Lyon (Rhone)

Dr J. Cohen Médecin chef, Hôpital psychiatrique de Mareville, Laxou, près

Nancy (Meurthe-et-Moselle)

Mile le Dr A. Deschamps Médecin chef, Hôpital psychiatrique de Fleury-les-Aubrais, près

Orleans (Loiret)

Dr P. Dubor Médecin des Hôpitaux psychiatriques, 46, Rue des Trois-Pierres,

Lyon (Rhone)

Dr R. EbtingerService de clinique psychiatrique de ГHôpital de Strasbourg (Bas-

Rhin) 80, Avenue des Vosges

Prof. R. Fau Chef du service de neuro-psychiatrie, Hôpital de Grenoble (Isère)

Dr M. Favier Médecin Commandant, Médecin chef du Service de neuro-

psychiatrie de ГHôpital militaire Desgenettes, Lyon (Rhone)

Dr P. Fournial Médecin chef de ГHôpital psychiatrique de Cadillac (Gironde)

Mme le Dr J. Gayral 25, Allées des Demoiselles, Toulouse (Haute-Garonne)

195

Dr J. Grambert Médecin chef de ГHôpital psychiatrique Sainte-Marie, Privas

(Ardèche)

Prof, agrégé J. Guyotat Professeur à la Faculté de médecine de Lyon, 11, Q $\mu$ ai du General

Sarrail, Lyon (Rhone)

Dr M. Henne Médecin chef de ГHôpital psychiatrique de Saint-Venant (Pas-de-

Calais)

Dr M. Houser Médecin chef de ГHôpital psychiatrique Sainte-Madeleine, Bourg 
(Ain)

Prof, agrégé L. Israel Assistant du Service de clinique psychiatrique, 6, Avenue de la

Forêt Noire, Strasbourg (Bas-Rhin)

Dr P. Koetschet Société des usines chimiques Rhône-Ponlenc, Paris

Dr P. Lambert Médecin chef de ГHôpital psychiatrique de Bassens (Savoie)

Dr R. Lanter Médecin chef de ГHôpital psychiatrique de RoufГach (Haut-Rhin)

Dr M. Lauxerois Médecin chef de $\Gamma$ Hôpital psychiatrique Sainte-Marie, Clermont-

Ferrand (Puy-de-Dôme)

Mile le Dr T. Lempérière Chef de clinique du Service de clinique des maladies mentales de ГHôpital Sainte-Anne, 42, Rue Henri-Barbusse, Paris, $5^{\circ}$

Dr A. Marin Chef de clinique du Service de clinique neuro-psychiatrique et Hygiene mentale, Hôpital du Vinatier, Bron (Rhone)

Dr H. Maurel Médecin chef, Hôpital psychiatrique de Rouffach (Haut-Rhin)

Dr A. Mestrallet Médecin chef, Hôpital psychiatrique St-Jean-de-Dieu, Lyon

(Rhone)

Dr H. Mignot Médecin chef, Hôpital psychiatrique de Maison-Blanche,

Neuilly-sur-Marne (Seine-et-Oise)

Dr R. Millon Médecin chef, Hôpital psychiatrique de Saint-Egreve (Isère)

M. R. Morice Société Parisienne d'Expansion chimique, Specia, Paris

M. R. Pannier Société Parisienne d'Expansion chimique, Specia, Paris

Dr J. Pellerat 53, Chemin de Tassin, Tassin-la-demi-Lune (Rhone)

Dr J. Perrin Médecin chef, Hôpital psychiatrique de Rouñ $1 / 8$ ch (Haut-Rhin)

Prof. B. Pommé Chef du Service clinique neuro-psychiatrique, Hôpital general,

Clermont-Ferrand (Puy-de-Dôme)

Prof. L. Revol Pharmacien-chef de ГHôpital psychiatrique du Vinatier, Bron

(Rhone)

Dr R. Reyss-Brion Chef de clinique du Service clinique de neuro-psychiatrie, Hôpital psychiatrique du Vinatier, Bron (Rhone)

Mile le Dr M. Rives 53, Avenue Berthelot, Lyon (Rhone)

Dr J. Schneider Assistant à $\quad$ Hôpital Pasteur, Colmar (Haut-Rhin)

196

Prof, agrégé B. Schott Chef-Adjoint du Service de clinique de neuro-psychiatrie à

l'Hôpital Edouard Herriot, Lyon (Rhone)

Dr L. Singer Médecin des Hôpitaux de Strasbourg, 1, Place de l'Homme de Fer

Strasbourg (Bas-Rhin)

Mile le Dr H. Stern Assistante à ГHôpital de Toulouse, 22, Allées F. Verdier, Toulouse

(Haute-Garonne)

Dr H. Vermorel Médecin chef, Hôpital psychiatrique de Lorquin (Moselle) 